\title{
EDITORIALS
}

\section{SURGICAL MANAGEMENT OF CORNEAL DISEASE}

It seems to have become an almost knee-jerk response for corneal surgeons to treat corneal pathology with penetrating keratoplasty. It is perhaps understandable how this dependence on penetrating keratoplasty has come about. After all it is a simple operation, and with good-quality corneal tissue available on demand through today's excellent eye bank network the whole procedure is almost too easy. No one needs to worry about tissue matching as the American multicentre trial has shown it to be of no benefit! ${ }^{1}$ The short-term results of penetrating keratoplasty look favourable and we know that the risk of rejection declines with time. But in the outpatient clinic all is not as well as some of the literature might have us believe, and a recent report from the USA cites the single largest current indication for penetrating keratoplasty as being regrafting for graft failure. ${ }^{2}$ Long-term follow-up indicates that grafts are failing not only from rejection but from endothelial depletion and recurrence of disease.

The paper in this issue by McLean et $a l^{3}$ on the treatment of pellucid marginal degeneration by simple wedge incision is thus a welcome breath of fresh air. However, even these authors put forward penetrating keratoplasty as their treatment of second choice, despite conceding that large eccentrically placed penetrating grafts have a higher risk of rejection than the low rates generally reported in penetrating keratoplasty for keratoconus. They also make mention of other possible techniques for treating pellucid marginal degeneration, such as local lamellar excision or, alternatively, epikeratoplasty. Yet surely these procedures are clearly preferable to a penetrating graft in the management of pellucid marginal degeneration and indeed for all conditions of corneal ectasia or corneal pathology not affecting the corneal endothelium?

Wedge excision for pellucid marginal degeneration can be viewed as a purely keratorefractive procedure. It might be argued that it is also prophylactic against the development of complications such as acute hydrops, although its late occurrence in one case following wedge excision would tend to negate this. As a keratorefractive procedure its effectiveness must of course be assessed in terms of visual acuity and refraction. In McLean's series the post-operative best corrected acuities 'at some stage' were all better than $6 / 9$, which would suggest a successful outcome. The long-term analysis of refractive outcome in groups of patients having keratorefractive surgery has been much enhanced by the development of vector analysis and vector decomposition by Olsen and Dam-Johansen. ${ }^{4}$ In this series of patients there was a long-term astigmatic drift with a tendency for development of increasing against-the-rule astigmatism. This pattern of astigmatism is that which occurs in the primary disease process, so could be regarded as either a regression of effect of the surgical procedure, a progression of the disease process, or a combination of both these factors.

One of the strengths of the paper of McLean et al. is the length of follow-up - ranging from 1 to 10 years with a mean follow-up of over 5 years. In this series both 10/0 nylon and 10/0 polypropylene (Prolene) sutures were used, with selective suture removal undertaken in an attempt to achieve optimal refractive outcome. Nylon has been used for many years and is easier to handle, but its wound tensioning effect is lost within a year through biodegradation, and if problems from suture breakup are to be avoided all sutures must be electively removed. Polypropylene and polyester (Mersilene) are essentially non-biodegradable, and are probably the ideal sutures for this type of surgery, although some have argued that polyester produces a greater tissue reaction than nylon and as such is unsuitable for penetrating keratoplasty. ${ }^{5}$

Unfortunately we do not have a clear picture from this study of the possible merits of prolonged suture retention on the evolution of astigmatism in this condition. There was long-term astigmatic drift of quite a high order in some of the patients in this series, so there is no doubt that further research is required to define the optimal management path for these patients. 
Whilst pure pellucid marginal degeneration is a relatively rare corneal ectasia, it can be viewed as part of a spectrum of disease including keratoconus and keratoglobus. It is hoped that this article may stimulate thought and possible change in practice for surgeons tackling these problems. Unfortunately in these days of active cost-cutting in health care it has become increasingly difficult to obtain data on longterm follow-up of the results of treatment for these conditions, but it is only through collection and documentation of this information that we can rationally choose the appropriate treatment for patients who are looking for more than a shortterm solution to their condition.

St George's Hospital

London

\section{References}

1. The Collaborative Corneal Transplantation Studies Research Group. The Collaborative Corneal Transplantation Studies (CCTS). Effectiveness of histocompatibility matching in high-risk corneal transplantation. Arch Ophthalmol 1992;110:1392-403.

2. Flowers CW, Chang KY, McLeod SD, Irvine JA, McDonnell PJ, Rao N, Smith RE. Changing indications for penetrating keratoplasty, 1989-1993. Cornea 1995; 14:583-8.

3. McLean H, Robinson LP, Weschler AW. Long-term results of corneal wedge excision for pellucid marginal degeneration. Eye 1997;11:613-7.

4. Olsen T, Dam-Johansen M. Evaluating surgically induced astigmatism. J Cataract Refract Surg 1994; 20:517-22.

5. Bradley AB, Drews-Botch C, Gemmill M, Guell J, Murad M, Waring GO. Complications of Mersilene sutures in penetrating keratoplasty. Refract Corneal Surg 1992;8:296-305.

\section{PREVENTING VISUAL IMPAIRMENT IN PRE-TERM BABIES}

If it is the business of an ophthalmologist to prevent blindness, it must be the business of a paediatric ophthalmologist to prevent childhood blindness. In the UK most of the causes of childhood blindness are not easily preventable, but one group - premature babies - deserves our attention because they are at much higher risk of visual impairment than their peers and it is likely that much of this is preventable. Premature infants are at risk of visual impairment from retinopathy of prematurity (ROP), nystagmus, cortical problems, and high myopia. ${ }^{1}$ They are also at high risk of strabismus.

Primary prevention of these problems would be by lowering the incidence of prematurity, but there is little evidence that this is occurring. The rise in assisted conception, which appears to predispose to prematurity and to $\mathrm{ROP},{ }^{2}$ is likely to increase the incidence in the UK, and ROP appears to be a growing problem worldwide.

The paper by Pennefather et al. in this issue $e^{3}$ is the most comprehensive study of the long-term refraction of premature infants yet done. It seems from this study that the myopia associated with prematurity is itself the result of ROP and not of the prematurity per se. Some of this myopia is likely to be due to the effects of cryotherapy treatment for the ROP.

It is almost 10 years since the first published results of the Cryo-ROP study, ${ }^{4}$ which clearly showed that retinal cryotherapy could reduce the incidence of blindness in children with ROP. Following this, mass screening programmes have been initiated with the intention of treating at-risk infants. In this period laser retinal photocoagulation has become popular with many ophthalmologists since it seemed to produce less immediate problems than did cryotherapy. The indirect delivery system of first the argon laser and, more recently, the diode laser, has meant that it is quite feasible to laser the peripheral retina in pre-term babies, and this treatment has not only been shown to result in less myopia than cryotherapy ${ }^{5}$ but may also be superior to cryotherapy in its ability to control the disease. ${ }^{6}$

Although laser photocoagulation can be delivered with topical anaesthesia, it is probably better to give the infant a general anaesthetic since this not only gives control over ventilation but also provides adequate analgesia. A recent study has confirmed the long-held suspicion that invasive procedures performed on neonates without analgesia can produce long-lasting adverse psychological effects. ${ }^{7}$

Despite this improvement in the treatment of cicatricial ROP, the visual results following treatment are not good. For example, in the study by Pennefather et al., retinal detachment occurred in 4 of the 27 eyes that developed cicatricial ROP, and 8 of these 27 had not been screened for the development of ROP. In White and Repka's paper, ${ }^{5}$ of the 12 eyes treated with laser only one had an acuity recordable as $6 / 6$ and 4 had myopia of over 10 dioptres. These results are not unusual, and would fit with most surgeons' experience. Can we do better? If Pennefather et al. were to repeat their studies in 10 years' time would the incidence of retinal detachment and myopia be lower?

The original treatment threshold was set in the Cryo-ROP study as being that degree of ROP at which roughly $50 \%$ of the patients could be expected to progress to blindness, and this was done for the 